\title{
To Infinity and Beyond: SEM to X-Ray Fluorescence Imaging to Determine Micronutrients in Helicobacter pylori.
}

\author{
L-M. Joubert*, M.R. Amieva**, S. Tan**, S. Webb***, B.Lai**** \\ * CSIF, Beckman Center, Stanford University Medical School, Stanford CA 94305 \\ ** Department Microbiology and Immunology, Stanford University, Stanford CA 94305 \\ *** Stanford Synchrotron Radiation Lightsource, 2575 Sand Hill Rd, Menlo Park, CA, 94025 \\ **** Advanced Photon Source, Argonne National Laboratory, Argonne, Chicago, IL 60439
}

Helicobacter pylori $(\mathrm{Hp})$ is a bacterium that infects the human stomach, establishing intimate contacts with gastric mucous cells, and increasing the risk for gastro-duodenal ulcers and gastric cancer [1]. The more virulent strains of $H p$ use a macromolecular 'syringe' that injects bacterial proteins (cagA) into the host cell [2,3]. Recent physiological results suggest a capacity of $H p$ to extract iron from the surrounding epithelial cells upon attachment and expression of cagA effector protein, whereupon cell polarity is altered [4]. Iron absorption [5,6], and probably also Nickel and Zinc, seem to be intimately involved in the successful colonization of the host epithelial cell.

Extensive Fluorescence and SEM investigation [4] revealed colonization features of $H p$ WT versus cagA mutants (lacking the effector protein). To determine which micronutrients are acquired by $H p$ from the host cells for successful colonization of its replicative niche, we apply synchrotron X-ray fluorescence (XRF) microanalytical techniques at Beamline 2-3 (SSRL) and 2-IDD (APS, Argonne). Parallel experiments with $H p$ WT and CagA mutant cells are performed at different time points (5min, 24h, 48h) to investigate micronutrient (Fe, Ni, Zn) concentration in bacterial microcolonies attached to MDCK cells grown on Transwell ${ }^{\mathrm{TM}}$ filters, using techniques recently described [7]. To precisely localize $\mathrm{Hp}$ microcolonies on MDCK cells for high-resolution XRF scans, we additionally immunolabel $H p$ colonies with anti-Hp polyclonal primary and $15 \mathrm{~nm}$ gold-conjugated secondary antibodies. Nanogold particles attached to the bacterial surface can be visualized by Field Emission SEM (backscatter detection)(Fig.2A), followed by XRF detection (Fig.2B) of the same microcolonies to optimize high-resolution scanning times with smaller spot (step) sizes and longer dwell (live) times. To retain epitope activity, primary fixation is done with $2 \%$ PFA, and glutaraldehyde (4\%) added as fixative only after immunogold labeling has been completed. To avoid contamination and redistribution of elements only glass distilled EM grade chemicals and no surfactants are used, and cells are critical point dried after ethanol dehydration. To avoid interference with microbeam analysis, osmication is avoided, and gold-sputtering is replaced by carbon-coating for high resolution FESEM. Filter segments $(4 \mathrm{x} 4 \mathrm{~mm})$ are transferred to $\mathrm{Si}_{3} \mathrm{~N}_{4}$ windows $(200 \mathrm{~nm}$ thick, 5x5mm frame), and Molybdenum TEM grids (200mesh) additionally superimposed on $\mathrm{Si}_{3} \mathrm{~N}_{4}$ windows to correlate microcolony position (X-Y coordinates) between SEM and XRF analysis.

XRF scans of large surface areas $(100 \times 100 \mu \mathrm{m}$, spot size $1 \mu \mathrm{m}$, dwell time $0.8 \mathrm{sec})$ at BL2-3 showed low-resolution micronutrient features of $\mathrm{Hp}$, while also suggesting differences between WT and cagA mutants. Subsequent high resolution scans at beamline 2-IDD, of smaller $(10 x 10 \mu \mathrm{m}$, step size $0.1 \mu \mathrm{m}$, live time $2 \mathrm{sec}$ ) of gold-labeled areas revealed a striking correlation of micronutrients with microcolony position (Fig.3), and further differences between WT and cagA mutants at different timepoints. Further quantification, matching microcolony shape and volume with XRF maps, is in progress. 
References

[1] M.R. Amieva et al., Science 300 (2003) 1430.

[2] S. Backert et al., Cell Microbiol 2 (2000) 155.

[3] S. Odenbreit et al. Science 287(2000) 1497.

[4] S. Tan et al., PLoS Pathog. 5(2009)p.e 1000407

[5] M.A. Fischbach et al. Nat Chem Biol 2 (2006) 132.

[6] A. Barabino. Helicobacter 7 (2002) 71.

[7] K.M. Kemner et al., Science 306 (2004) 686.
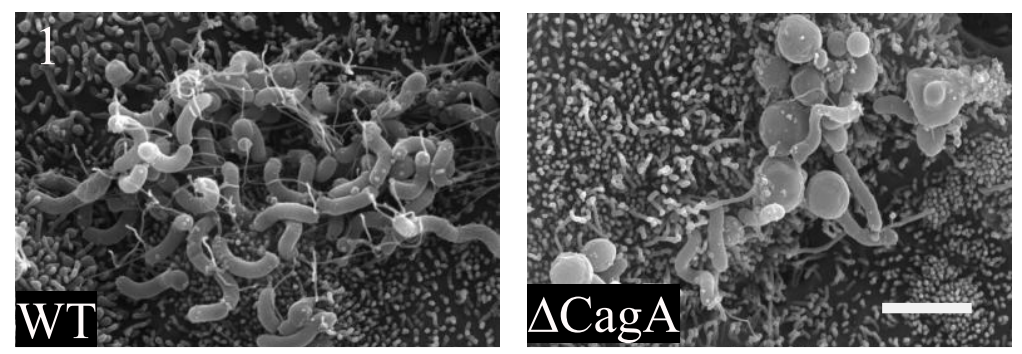

FIG.1. SEM images of $H p$ microcolonies (1d) on apical surface of polarized MDCK cells on Transwell filters $($ scalebar $=2 \mathrm{um})$

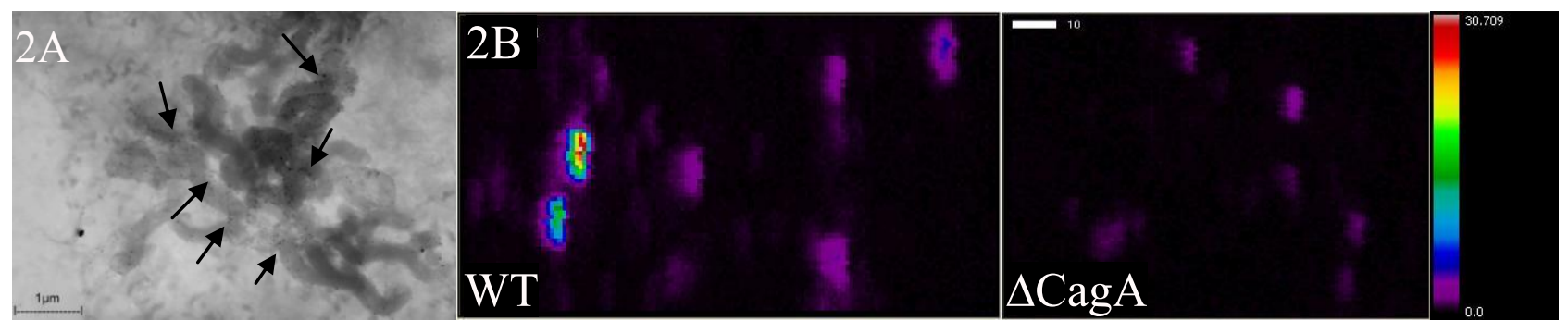

FIG.2 (A) FESEM image (BSE) of 15nm gold-labeled (arrows) $H p$ microcolony (WT, 1d)

(B) XRF maps at BL2-3 indicating Fe concentration of gold-labeled $H p$ microcolonies (WT 2d; cagA 2d). Scale prism correlates color with concentration.

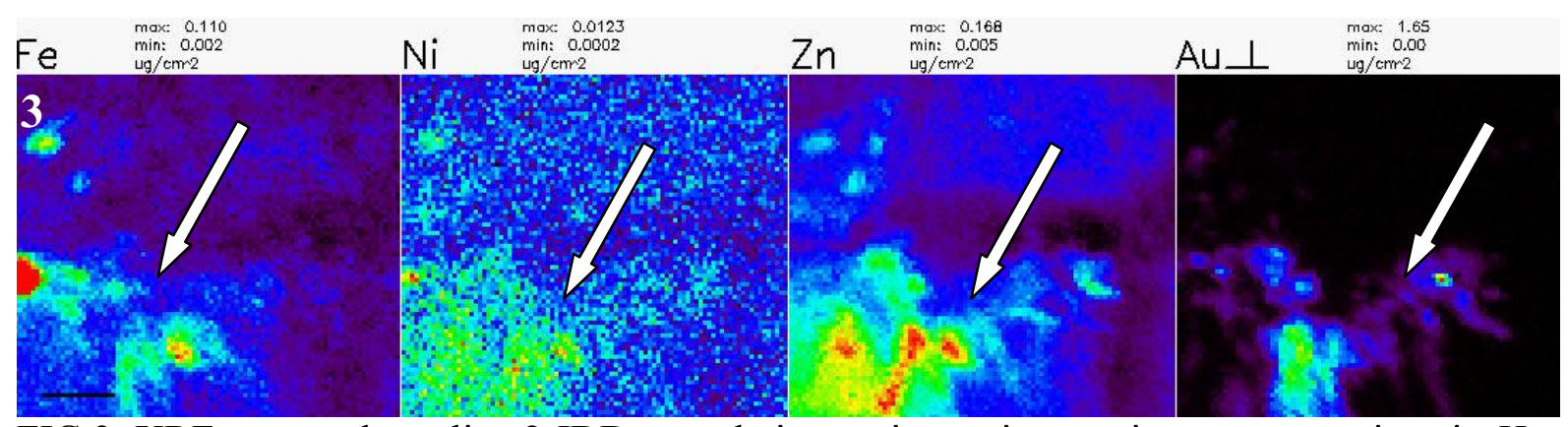

FIG.3: XRF maps at beamline 2-IDD, correlating various micronutrient concentrations in $H p$ microcolonies, with the Au signal localizing bacteria in a single scan (WT 2d) (scalebar $=2 \mathrm{um}$ ) 